\title{
Determining Replenishment Lot Size and Shipment Policy for an EPQ Inventory Model with Delivery and Rework
}

\author{
Leopoldo Eduardo Cárdenas-Barrón, ${ }^{1}$ Gerardo Treviño-Garza, ${ }^{2}$ \\ Ata Allah Taleizadeh, ${ }^{3}$ and Pandian Vasant ${ }^{4}$ \\ ${ }^{1}$ School of Engineering and Sciences, Tecnológico de Monterrey, Eugenio Garza Sada 2501 Sur, CP 64849, Monterrey, NL, Mexico \\ ${ }^{2}$ BNSF Railway Company, 2650 Lou Menk Drive, Fort Worth, TX 76131-2830, USA \\ ${ }^{3}$ Department of Industrial Engineering, University of Tehran, Tehran 41764411, Iran \\ ${ }^{4}$ Department of Fundamental and Applied Sciences, Universiti Teknologi PETRONAS, 32610 Perak, Malaysia
}

Correspondence should be addressed to Leopoldo Eduardo Cárdenas-Barrón; lecarden@itesm.mx

Received 27 November 2014; Revised 13 June 2015; Accepted 15 June 2015

Academic Editor: Yann Favennec

Copyright (C) 2015 Leopoldo Eduardo Cárdenas-Barrón et al. This is an open access article distributed under the Creative Commons Attribution License, which permits unrestricted use, distribution, and reproduction in any medium, provided the original work is properly cited.

\begin{abstract}
The determination of production-shipment policies for a vendor-buyer system is dealt within this paper. The main objective is to derive the optimal replenishment lot size and shipment policy for an EPQ inventory model with multiple deliveries and rework. This inventory model contains two decision variables: the replenishment lot size and the number of deliveries. Previous researches solve this inventory model considering both variables to be continuous. However, the number of deliveries must be considered as a discrete variable. In this direction, this paper solves the inventory model considering two cases: Case 1: the replenishment lot size as a continuous variable and the number of shipments as a discrete variable and Case 2: the replenishment lot size and the number of shipments as discrete variables. The final results are two simple and easy-to-apply solution procedures to find the optimal values for the replenishment lot size and the number of deliveries for each case.
\end{abstract}

\section{Introduction}

A key challenge in the inventory management in any organization is to answer the following simple question: How many products to order? This question is responded to by the traditional economic order quantity (EOQ) inventory model. It is well known that the EOQ inventory model was derived by Harris [1] in 1913. Later, the economic production quantity (EPQ) inventory model was proposed by Taft [2] in 1918. It is worthy to mention that since then, several extensions to both inventory models have been derived by several scholars, that is, Taleizadeh et al. [3], Chen [4], and Pal et al. [5], just to name a few recent researches. In Cárdenas-Barrón et al. [6] Ford Whitman Harris is named as the founding father of the inventory theory.

Recently, Chiu et al. [7] and Chiu et al. [8] determine the optimal replenishment lot size and shipment policy for an EPQ inventory model with multiple deliveries and rework. We have read the papers by Chiu et al. [7] and Chiu et al. [8] with substantial interest and we have found that their inventory model contains two decision variables: the replenishment lot size and the number of deliveries. The works of Chiu et al. [7] and Chiu et al. [8] solve the inventory model considering both variables to be continuous. However, the number of deliveries must be considered to be a discrete variable. We think that the researchers that have been attracted by works of Chiu et al. [7] and Chiu et al. [8] may be interested in knowing the solution procedure that gives the optimal solution to the decision variables according to its nature. In this direction, this paper solves their inventory model considering two cases: Case 1: the replenishment lot size as a continuous variable and the number of shipments as a discrete variable and Case 2: the replenishment lot size and number of shipments as discrete variables. To solve both cases we use the algebraic method of completing perfect squares and the result of García-Laguna et al. [9]. The algebraic 
method of completing perfect squares has been used amply by many scholars since 1996 (Grubbström [10], Omar et al. [11], Wee et al. [12], and Yang and Wee [13]). Conversely, Chiu et al. [7] consider both decision variables to be continuous and then use the classical optimization technique (differential calculus) to determine the replenishment lot size and shipment policy for an extended EPQ model with delivery and quality assurance issues. It is important to point out that Chiu et al. [8] derive the same inventory problem of Chiu et al. [7] using the algebraic method of completing perfect squares and they also considered both variables to be continuous.

Recently, Treviño-Garza et al. [14] solve optimally a family of inventory models that deal with an EPQ for an integrated vendor-buyer system considering that the production system creates defective products. Taleizadeh et al. [15] propose EPQ inventory model with rework of defective items when multishipment policy is used. Their inventory model determines optimally the selling price, the lot size, and the number of shipments. Sana [16] develops an EOQ inventory model for conforming and nonconforming quality products in which the nonconforming products are sold at a reduced price. Pal et al. [17] derive an EPQ inventory model to determine the optimal buffer for a stochastic demand considering preventive maintenance. Pal et al. [18] propose an EPQ inventory model for an imperfect production system that takes into account that defective items are reworked after the regular production time. Das Roy et al. [19] develop an economic production lot size model for a manufacturing system that produces defective items. These defective items are accumulated and then reworked. This inventory model also permits shortages with partial and full backordering.

\section{Optimizing the Replenishment Lot Size and the Number of Shipments}

This section presents the optimizing procedure of the replenishment lot size and the number of shipments for the inventory problem given in Chiu et al. [7] and Chiu et al. [8].

2.1. The Replenishment Lot Size and Shipment Policy for an Extended EPQ Model with Delivery and Quality Assurance Issues (Chiu et al. [7] and Chiu et al. [8]). Chiu et al. [7] and Chiu et al. [8] derived the following long-run average costs $E[T C U(Q)]$ :

$$
\begin{aligned}
& E[T C U(Q)]=\frac{C \lambda}{(1-\varphi E[x])}+\frac{\left(K+n K_{1}\right) \lambda}{(1-\varphi E[x]) Q} \\
& +\frac{C_{R} E[x](1-\theta) \lambda}{(1-\varphi E[x])}+\frac{C_{S} E[x] \varphi \lambda}{(1-\varphi E[x])}+C_{T} \lambda \\
& +\frac{h Q \lambda}{2 P_{1}(1-\varphi E[x])}\left[\left(2 E[x]-(E[x])^{2}\right.\right. \\
& \left.\left.-\varphi(E[x])^{2}\right)(1-\theta)\right]+\frac{h Q \lambda}{2 P(1-\varphi E[x])}+\left(1-\frac{1}{n}\right) \\
& \quad \cdot\left[\frac{h Q(1-\varphi E[x])}{2}-\frac{h Q \lambda}{2 P}-\frac{h Q E[x](1-\theta) \lambda}{2 P_{1}}\right]
\end{aligned}
$$

$$
\begin{aligned}
& +\frac{h_{1}(E[x])^{2} Q \lambda(1-\theta)^{2}}{2 P_{1}(1-\varphi E[x])}+\left(\frac{1}{n}\right) \frac{h_{2} Q}{2}(1-\varphi E[x]) \\
& +\left(1-\frac{1}{n}\right) \frac{h_{2} Q \lambda}{2 P}+\frac{h_{2} Q}{2}\left[\left(1-\frac{1}{n}\right) \frac{E[x] \lambda(1-\theta)}{P_{1}}\right],
\end{aligned}
$$

where the notation is as follows.

Parameters

$C$ : production cost (\$/unit),

$C_{R}$ : rework cost (\$/unit),

$C_{S}$ : disposal cost per scrap product (\$/unit),

$C_{T}$ : delivery cost per product shipped to customers (\$/unit),

$K$ : setup cost per cycle (\$/cycle; \$/lot; \$/run),

$K_{1}$ : fixed delivery cost per shipment (\$/shipment),

$h$ : holding cost (\$/unit/time unit),

$h_{1}$ : holding cost for each reworked product (\$/unit/time unit),

$h_{2}$ : holding cost for each item kept by customer (\$/unit/time unit),

$\lambda$ : demand rate (units/time unit),

$P$ : production rate (units/time unit),

$P_{1}$ : reworking rate (units/time unit),

$x$ : a proportion of defective items; it is assumed to be a random variable with a known probability density,

$E(x)$ : expected value of $x$,

$\theta$ : a proportion of the imperfect quality products is scrap; it is assumed to be known and constant,

$\theta_{1}$ : a proportion of reworked products fails and becomes scrap; it is assumed to be known and constant,

$\varphi$ : overall scrap rate per cycle; $\varphi=\theta+(1-\theta) \theta_{1}$,

$U(a, b)$ : indicates uniform distribution with range $(a, b)$.

Variables

Q: the replenishment lot size (units),

$n$ : the number of shipments.

The detailed derivation of each term in $E[T C U(Q)]$ can be found in Chiu et al. [7]. Note that they consider only one variable $(Q)$. Conversely, this paper considers the long-run average costs with two decision variables: $Q$ and $n$, where $Q$ is a continuous variable and $n$ is a discrete variable. Hence $E[T C U(Q, n)]$ is rewritten as follows:

$$
E[T C U(Q, n)]=\mu_{1} Q+\frac{\mu_{2}}{Q}+\mu_{3},
$$


where the constants $\mu_{1}, \mu_{2}$, and $\mu_{3}$ are given by

$$
\begin{aligned}
\mu_{1} & =\frac{1}{2}\left\{\frac { \lambda } { ( 1 - \varphi E ( x ) ) } \left[h \left\{\left[\frac{E(x)}{P_{1}}\right][2-E(x)(1+\varphi)]\right.\right.\right. \\
\cdot & \left.\left.(1-\theta)+\frac{1}{P}\right\}+\frac{h_{1}[E(x)]^{2}(1-\theta)^{2}}{P_{1}}\right]+\left(h_{2}-h\right) \\
& \cdot\left[(1-\varphi E(x))-\lambda\left(\frac{1}{P}+\frac{E(x)(1-\theta)}{P_{1}}\right)\right]\left(\frac{1}{n}\right) \\
& +\left(h_{2}-h\right) \lambda\left(\frac{1}{P}+\frac{E(x)(1-\theta)}{P_{1}}\right)+h(1 \\
& -\varphi E(x))\}>0, \\
\mu_{2} & =\frac{\lambda\left(K+n K_{1}\right)}{(1-\varphi E(x))}>0, \\
\mu_{3} & =\left[\frac{\lambda}{(1-\varphi E(x))}\right]\left\{C+C_{T}+\left[C_{R}(1-\theta)+\varphi\left(C_{S}\right.\right.\right. \\
& \left.\left.\left.-C_{T}\right)\right] E(x)\right\} .
\end{aligned}
$$

It is important to remark that the total cost $E[T C U(Q, n)]$ is a mixed integer nonlinear optimization problem when the lot size $(Q)$ is a continuous variable and the number of shipments $(n)$ is a discrete variable. On the contrary, the total cost $E[T C U(Q, n)]$ is an integer nonlinear optimization problem when both variables $(Q$ and $n)$ are considered to be discrete variables.

To optimize the total cost $E[T C U(Q, n)]$ a sequential optimization procedure of two stages is applied. First stage optimizes the replenishment lot size $(Q)$ by the algebraic method of completing perfect squares (see, e.g., CárdenasBarrón [20]). Second stage optimizes the number of shipments $(n)$ using the result of García-Laguna et al. [9].

Stage I (Optimizing the Replenishment Lot Size (Q)). In the research work of Cárdenas-Barrón [20] it was demonstrated by the algebraic method of completing perfect squares that a function of type $\mu_{1} Q+\mu_{2} / Q$ is always minimized for $Q=$ $\sqrt{\mu_{2} / \mu_{1}}$, which attains the minimum at $f(Q)=2 \sqrt{\mu_{1} \mu_{2}}$ where $Q$ is a continuous variable and $\mu_{1}$ and $\mu_{2}$ are both greater than zero. Consequently, the replenishment lot size (Q) is

$$
\begin{aligned}
Q & =\sqrt{\frac{\mu_{2}}{\mu_{1}}}=\left(2 \lambda\left(K+n K_{1}\right)\right. \\
& \cdot\left(\lambda \left[h\left\{\left[\frac{E(x)}{P_{1}}\right][2-E(x)(1+\varphi)](1-\theta)+\frac{1}{P}\right\}\right.\right. \\
& \left.+\frac{h_{1}[E(x)]^{2}(1-\theta)^{2}}{P_{1}}\right]+(1-\varphi E(x))\left\{\left(h_{2}-h\right)\right. \\
& \cdot\left[(1-\varphi E(x))-\lambda\left(\frac{1}{P}+\frac{E(x)(1-\theta)}{P_{1}}\right)\right]\left(\frac{1}{n}\right)
\end{aligned}
$$

$$
\begin{aligned}
& +\left(h_{2}-h\right) \lambda\left(\frac{1}{P}+\frac{E(x)(1-\theta)}{P_{1}}\right) \\
& \left.+h(1-\varphi E(x))\})^{-1}\right)^{1 / 2} .
\end{aligned}
$$

And the minimal total cost is

$$
\begin{aligned}
& E[T C U(n)]=2 \sqrt{\mu_{1} \mu_{2}}+\mu_{3} \\
& =2\left(\frac { 1 } { 2 } \left\{\frac { h \lambda } { ( 1 - \varphi E ( x ) ) } \left[\left[\frac{E(x)}{P_{1}}\right]\right.\right.\right. \\
& \left.\cdot[2-E(x)(1+\varphi)](1-\theta)+\frac{1}{P}\right]+\left(h_{2}-h\right) \\
& \cdot\left[(1-\varphi E(x))-\lambda\left(\frac{1}{P}+\frac{E(x)(1-\theta)}{P_{1}}\right)\right]\left(\frac{1}{n}\right) \\
& +\frac{\lambda h_{1}[E(x)]^{2}(1-\theta)^{2}}{(1-\varphi E(x)) P_{1}}+\left(h_{2}-h\right) \lambda\left(\frac{1}{P}\right. \\
& \left.\left.+\frac{E(x)(1-\theta)}{P_{1}}\right)+h(1-\varphi E(x))\right\} \\
& \left.\cdot\left[\frac{\lambda\left(K+n K_{1}\right)}{(1-\varphi E(x))}\right]\right)^{1 / 2}+\mu_{3} \\
& =\sqrt{\frac{2 \lambda}{(1-\varphi E(x))}}\left(\left\{\frac { h \lambda } { ( 1 - \varphi E ( x ) ) } \left[\left[\frac{E(x)}{P_{1}}\right]\right.\right.\right. \\
& \left.\cdot[2-E(x)(1+\varphi)](1-\theta)+\frac{1}{P}\right]+\left(h_{2}-h\right) \\
& \cdot\left[(1-\varphi E(x))-\lambda\left(\frac{1}{P}+\frac{E(x)(1-\theta)}{P_{1}}\right)\right]\left(\frac{1}{n}\right) \\
& +\frac{\lambda h_{1}[E(x)]^{2}(1-\theta)^{2}}{(1-\varphi E(x)) P_{1}}+\left(h_{2}-h\right) \lambda\left(\frac{1}{P}\right. \\
& \left.\left.+\frac{E(x)(1-\theta)}{P_{1}}\right)+h(1-\varphi E(x))\right\}[(K \\
& \left.\left.\left.+n K_{1}\right)\right]\right)^{1 / 2}+\mu_{3}=\sqrt{\frac{2 \lambda}{(1-\varphi E(x))}} \sqrt{f_{n}+\mu_{6}}+\mu_{3},
\end{aligned}
$$

where

$$
\begin{aligned}
f_{n} & =K_{1} \mu_{4} n+\frac{K \mu_{5}}{n}, \\
\mu_{4} & =\left(\frac{1}{(1-\varphi E(x))}\right) \\
& \cdot\left[h \lambda E(x)\left(\frac{\varphi}{P}+\frac{(1-E(x))(1-\theta)}{P_{1}}\right)\right. \\
& +h_{2} \lambda(1-\varphi E(x))\left(\frac{1}{P}+\frac{E(x)(1-\theta)}{P_{1}}\right) \\
& \left.+h(1-\varphi E(x))^{2}+\frac{h_{1} \lambda[E(x)]^{2}(1-\theta)^{2}}{P_{1}}\right]>0,
\end{aligned}
$$




$$
\begin{aligned}
\mu_{5} & =\left(h_{2}-h\right)\left[(1-\varphi E(x))-\lambda\left(\frac{1}{P}+\frac{E(x)(1-\theta)}{P_{1}}\right)\right], \\
\mu_{6} & =\left(\frac{K}{(1-\varphi E(x))}\right) \\
& \cdot\left[h \lambda E(x)\left(\frac{\varphi}{P}+\frac{(1-E(x))(1-\theta)}{P_{1}}\right)\right. \\
& +h_{2} \lambda(1-\varphi E(x))\left(\frac{1}{P}+\frac{E(x)(1-\theta)}{P_{1}}\right) \\
& \left.+h(1-\varphi E(x))^{2}+\frac{h_{1} \lambda[E(x)]^{2}(1-\theta)^{2}}{P_{1}}\right] \\
& +K_{1}\left(h_{2}-h\right)[(1-\varphi E(x)) \\
& \left.-\lambda\left(\frac{1}{P}+\frac{E(x)(1-\theta)}{P_{1}}\right)\right] .
\end{aligned}
$$

It is worthy to mention that the function $f_{n}$ has the same mathematical form $\gamma_{1} n+\gamma_{2} / n$ but in this function the decision variable $n$ is discrete, and obviously $n$ must be greater or equal than one. In the work of García-Laguna et al. [9] it was showed that when $n$ is discrete and $\gamma_{1}$ and $\gamma_{2}$ are greater than zero, then function of type $\gamma_{1} n+\gamma_{2} / n$ attains its minimum when $n$ is given as follows:

$$
n=\left\lceil-0.5+\sqrt{0.25+\frac{\gamma_{2}}{\gamma_{1}}}\right\rceil
$$

or

$$
n=\left\lfloor 0.5+\sqrt{0.25+\frac{\gamma_{2}}{\gamma_{1}}}\right\rfloor .
$$

It is important to remember that $\lceil\omega\rceil$ and $\lfloor\omega\rfloor$ are the smallest integer greater than or equal to $\omega$ and the largest integer less than or equal to $\omega$, respectively. Clearly, it is easy to see that $\lceil\omega\rceil=\lfloor\omega+1\rfloor$ if and only if $\omega$ is not a discrete value. In this circumstance the minimization problem has a single solution for $n$ which is $n^{*}=n$ (given by either of the two expressions in (7) and (8)). Otherwise, the minimization problem has two solutions for $n$ that are $n^{*}=n$ and $n^{*}=n+1$.

Considering the result of (7) and (8), then the solution to the discrete variable $(n)$ is as follows:

$$
n=\left\lceil-0.5+\sqrt{0.25+\frac{K \mu_{5}}{K_{1} \mu_{4}}}\right\rceil
$$

or

$$
n=\left\lfloor 0.5+\sqrt{0.25+\frac{K \mu_{5}}{K_{1} \mu_{4}}}\right\rfloor .
$$

It is obvious that $\mu_{4}>0$ and therefore $\gamma_{4}=K_{1} \mu_{4}$ is always greater than zero. However, $\gamma_{5}=K \mu_{5}$ can be positive, zero, or negative because the following term $\left(h_{2}-h\right)[(1-\varphi E(x))-$ $\left.\lambda\left(1 / P+E(x)(1-\theta) / P_{1}\right)\right]$ can be positive, zero, or negative. When $\gamma_{5}$ takes positive values then the optimal solution for $n$ is given by (9) or (10). On the contrary, for zero and negative values of $\gamma_{5}$, it is easy to see that $\gamma_{1} n+\gamma_{2} / n$ attains its global minimum value at $n=1$.

In many situations of the real life the replenishment lot size $(Q)$ could be an integral value too. If we constrain the replenishment lot size to be a discrete variable then applying the previous result $((7)$ and $(8))$ the replenishment lot size $(Q)$ is given by

$$
\begin{aligned}
Q & =\left[-0.5+\left(0.25+2 \lambda\left(K+n K_{1}\right)\left(\lambda\left[h\left\{\left[\frac{E(x)}{P_{1}}\right][2-E(x)(1+\varphi)](1-\theta)+\frac{1}{P}\right\}+\frac{h_{1}[E(x)]^{2}(1-\theta)^{2}}{P_{1}}\right]\right.\right.\right. \\
& +(1-\varphi E(x)) \\
& \left.\left.\left.\cdot\left\{\left(h_{2}-h\right)\left[(1-\varphi E(x))-\lambda\left(\frac{1}{P}+\frac{E(x)(1-\theta)}{P_{1}}\right)\right]\left(\frac{1}{n}\right)+\left(h_{2}-h\right) \lambda\left(\frac{1}{P}+\frac{E(x)(1-\theta)}{P_{1}}\right)+h(1-\varphi E(x))\right\}\right)^{-1}\right)^{1 / 2}\right]
\end{aligned}
$$

or

$$
\begin{aligned}
Q & =\left\lfloor 0.5+\left(0.25+2 \lambda\left(K+n K_{1}\right)\left(\lambda\left[h\left\{\left[\frac{E(x)}{P_{1}}\right][2-E(x)(1+\varphi)](1-\theta)+\frac{1}{P}\right\}+\frac{h_{1}[E(x)]^{2}(1-\theta)^{2}}{P_{1}}\right]+(1-\varphi E(x))\right.\right.\right. \\
& \left.\left.\left.\cdot\left\{\left(h_{2}-h\right)\left[(1-\varphi E(x))-\lambda\left(\frac{1}{P}+\frac{E(x)(1-\theta)}{P_{1}}\right)\right]\left(\frac{1}{n}\right)+\left(h_{2}-h\right) \lambda\left(\frac{1}{P}+\frac{E(x)(1-\theta)}{P_{1}}\right)+h(1-\varphi E(x))\right\}\right)^{-1}\right)^{1 / 2}\right\rfloor .
\end{aligned}
$$


A lower bound for the total cost $E[T C U(Q)]$ can be obtained straightforwardly. The lower bound is derived just considering both variables ( $Q$ and $n$ ) to be continuous variables. Therefore, the lower bound is given by

$$
\begin{aligned}
\mathrm{LB} & =\left[\sqrt{\frac{2 \lambda}{(1-\varphi E(x))}}\right] \\
& \cdot\left(\sqrt{K_{1}\left(h_{2}-h\right)\left[(1-\varphi E(x))-\lambda\left(\frac{1}{P}+\frac{E(x)(1-\theta)}{P_{1}}\right)\right]}\right. \\
& +\left(\frac { K } { ( 1 - \varphi E ( x ) ) } \left[h \lambda E(x)\left(\frac{\varphi}{P}+\frac{(1-E(x))(1-\theta)}{P_{1}}\right)\right.\right. \\
& +h_{2} \lambda(1-\varphi E(x))\left(\frac{1}{P}+\frac{E(x)(1-\theta)}{P_{1}}\right) \\
& \left.\left.\left.+h(1-\varphi E(x))^{2}+\frac{h_{1} \lambda[E(x)]^{2}(1-\theta)^{2}}{P_{1}}\right]\right)^{1 / 2}\right)+\mu_{3} .
\end{aligned}
$$

The above lower bound is usable only when $\left(h_{2}-h\right)[(1-$ $\left.\varphi E(x))-\lambda\left(1 / P+E(x)(1-\theta) / P_{1}\right)\right]$ is greater or equal than zero. If $\left(h_{2}-h\right)\left[(1-\varphi E(x))-\lambda\left(1 / P+E(x)(1-\theta) / P_{1}\right)\right]$ is negative then the lower bound is just the $E[T C U(Q)]$ given by (2). It is important to mention that this lower bound (13) can be attained just for the case when $Q$ is a continuous variable and $n$ is a discrete variable if and only if $n=\sqrt{K \mu_{5} / K_{1} \mu_{4}}$ is an integer value. On the contrary, for the case when both $Q$ and $n$ are considered to be discrete variables then this lower bound (13) can be attained when the optimal solutions for both variables, $Q$ and $n$, are discrete values, in other words, when $Q$ given by (4) is a discrete value and at the same time $n$ given by $n=\sqrt{K \mu_{5} / K_{1} \mu_{4}}$ is a discrete value too. It is important to mention that the lower bound for the total cost obtained by (13) or (2) is just for Case 1. Obviously, the lower bound for the total cost for Case 2 is the total cost for Case 1 .

2.2. Solution Procedure to Find the Replenishment Lot Size (Q) and the Number of Shipments $(n)$. Here, two cases are considered: Case 1: the replenishment lot size $(Q)$ being continuous and the number of shipments $(n)$ being discrete and Case 2: the replenishment lot size $(Q)$ being discrete and the number of shipments $(n)$ being discrete. Based on the previous results that were obtained in Section 2.1, the following two solution procedures are proposed.

Case 1: The Replenishment Lot Size (Q) Being Continuous and the Number of Shipments (n) Being Discrete

\section{Solution Procedure for Case 1}

Step 1. Compute $\left(h_{2}-h\right)\left[(1-\varphi E(x))-\lambda\left(1 / P+E(x)(1-\theta) / P_{1}\right)\right]$. If $\left(h_{2}-h\right)\left[(1-\varphi E(x))-\lambda\left(1 / P+E(x)(1-\theta) / P_{1}\right)\right] \leq 0$ then set $n=1$ and compute $Q$ using (4) and go to Step 4; else go to Step 2.

Step 2. Compute the integral value for $n$.

If $-0.5+\sqrt{0.25+K \mu_{5} / K_{1} \mu_{4}}$ is not a discrete value then $n^{*}=n$.
Else, $n^{*}=n$ and $n^{*}=n+1$, where $n$ is computed using (9) or (10).

Step 3. Given the discrete value of $n$ then compute the continuous value for the lot size $Q$ using (4).

Step 4. Compute the total cost using (2) or (5).

It is important to remark that if there exist two solutions for $n$ then there are two optimal solutions for the inventory problem. For each solution of $n$ do Steps 3 and 4 and report the two optimal solutions. A flow diagram for solution procedure of Case 1 is given in Figure 1.

Case 2: The Replenishment Lot Size (Q) Being Discrete and the Number of Shipments ( $n$ ) Being Discrete

Solution Procedure for Case 2

Step 1. Compute $\left(h_{2}-h\right)\left[(1-\varphi E(x))-\lambda\left(1 / P+E(x)(1-\theta) / P_{1}\right)\right]$.

If $\left(h_{2}-h\right)\left[(1-\varphi E(x))-\lambda\left(1 / P+E(x)(1-\theta) / P_{1}\right)\right] \leq 0$ then set $n=1$ and compute $Q$ using (11) or (12) and go to Step 4; else go to Step 2.

Step 2. Compute the integral value for $n$.

$$
\text { If }-0.5+\sqrt{0.25+K \mu_{5} / K_{1} \mu_{4}} \text { is not a discrete value then }
$$$$
n^{*}=n \text {. }
$$

Else, $n^{*}=n$ and $n^{*}=n+1$, where $n$ is computed using (9) or (10).

Step 3. Given the discrete value of $n$ then compute the discrete value for the lot size $Q$ using (11) or (12).

Step 4. Compute total cost using (2).

Here, it is important to notice that if two solutions for $n$ exist then there are two solutions. For each solution of $n$ do Steps 3 and 4 and choose the solution with the minimal total cost. The flow diagram of the solution procedure for Case 2 is given in Figure 2.

The example of Chiu et al. [7] and Chiu et al. [8] is solved with the proposed solution procedures. The data for the example is shown in Table 1 and the solutions are given in Table 2 .

Now, we illustrate the situation when $\left(h_{2}-h\right)[(1-\varphi E(x))-$ $\left.\lambda\left(1 / P+E(x)(1-\theta) / P_{1}\right)\right]$ is lower and equal to zero. Consider that the value of $h$ is 98 and the other values for the parameters are the same as given in Table 1 . The value of $\left(h_{2}-h\right)[(1-$ $\left.\varphi E(x))-\lambda\left(1 / P+E(x)(1-\theta) / P_{1}\right)\right]$ is negative and it is equal to -12.53271429 . Then by applying the proposed solution procedures we obtain the following.

For Case 1: the replenishment lot size $(Q)$ being continuous and the number of shipments $(n)$ being discrete, the optimal solution is

$\mathrm{Q}=1110.748506 ; n=1$ and $E[T C U]=$ 519292.08791321. 


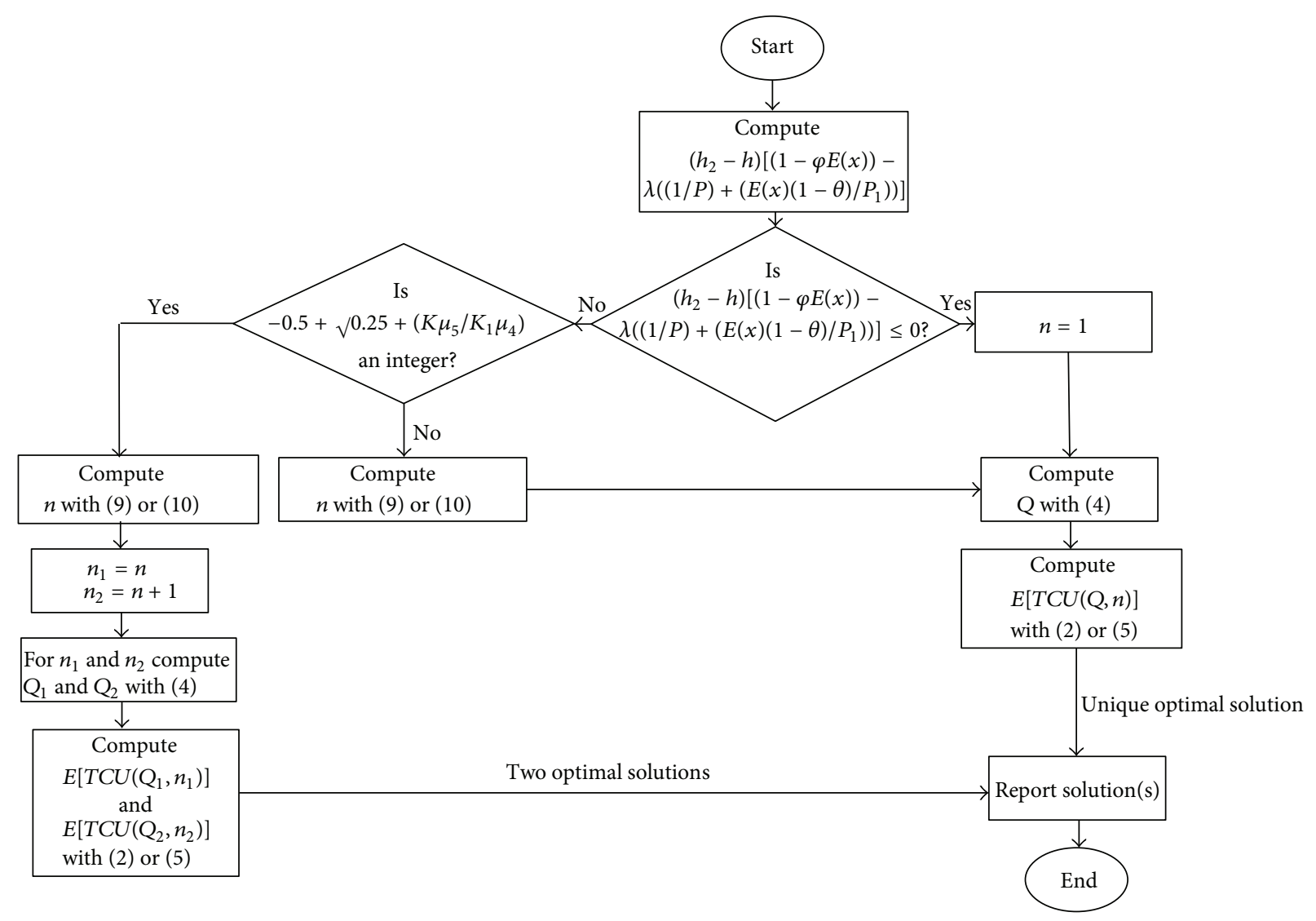

FIgURE 1: Flow diagram of solution procedure for Case 1.

For Case 2: the replenishment lot size $(Q)$ being discrete and the number of shipments $(n)$ being discrete, the optimal solution is

$$
Q=1111 ; n=1 \text { and } E[T C U]=548596.14413939 \text {. }
$$

\section{Discussion}

When applying the solution procedure for Case 1, we get the same solution as Chiu et al. [7] and Chiu et al. [8] but in a simple manner. On the other hand, the solution procedure of Chiu et al. [7] rounds the value of the number of shipments $(n)$. This action could provide us with a nonoptimal value for the number of shipments $(n)$. Although Chiu et al. [8] fix the previous mentioned shortcoming, their solution procedure requires to evaluate the total cost twice (one for each $n$ ) in order to compute the number of shipments $(n)$. Moreover, it is important to mention that Chiu et al. [7] and Chiu et al. [8] do not consider the situation when $\left(h_{2}-h\right)[(1-$ $\left.\varphi E(x))-\lambda\left(1 / P+E(x)(1-\theta) / P_{1}\right)\right] \leq 0$. Also, they do not solve the inventory problem when both variables are discrete. Furthermore, both solutions procedures proposed in this paper are easy to implement in a spreadsheet and do not require any computational effort. These are some important advantages that our paper has with respect to Chiu et al. [7] and Chiu et al. [8].
TABle 1: Data for the example.

\begin{tabular}{lc}
\hline Parameter & $\begin{array}{c}\text { From Chiu et al. [7] } \\
\text { and Chiu et al. [8] }\end{array}$ \\
\hline$C$ & 100 \\
$C_{R}$ & 60 \\
$C_{S}$ & 20 \\
$C_{T}$ & 0.1 \\
$K$ & 20000 \\
$K_{1}$ & 2000 \\
$h$ & 20 \\
$h_{1}$ & 40 \\
$h_{2}$ & 80 \\
$\lambda$ & 3400 \\
$P$ & 60000 \\
$P_{1}$ & 2100 \\
$x$ & Uniform $(0,0.3)$ \\
$E(x)$ & $0.15^{*}$ \\
$\theta$ & 0.1 \\
$\theta_{1}$ & 0.1 \\
$\varphi=\theta+(1-\theta) \theta_{1}$ & 0.19 \\
\hline
\end{tabular}

${ }^{*}$ Remember that for a uniform distribution with range $(a, b)$ the expected value is defined as $E(x)=(a+b) / 2$. 


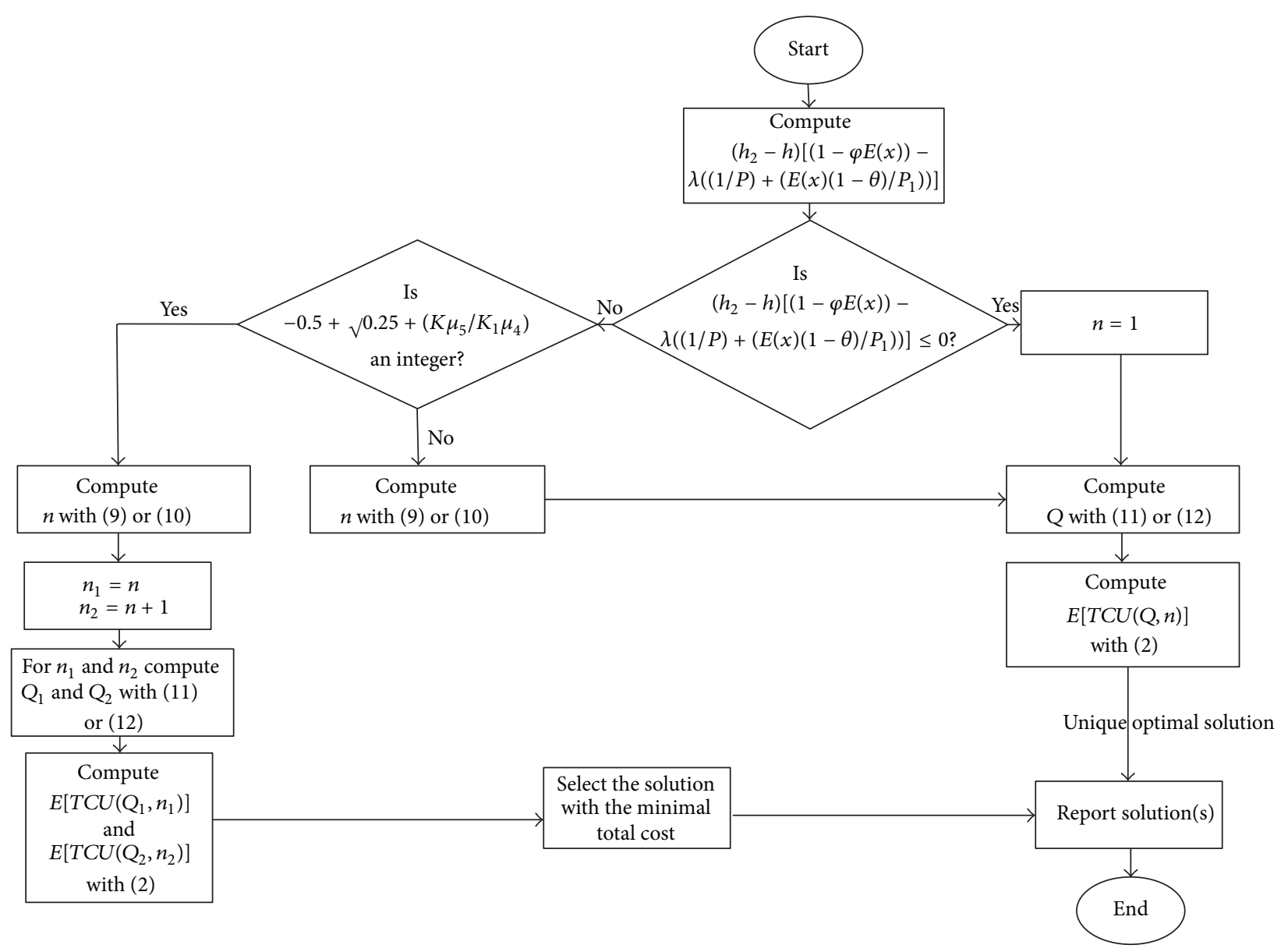

FIGURE 2: Flow diagram of solution procedure for Case 2.

TABLE 2: Results for the example.

\begin{tabular}{lccc}
\hline Instance & $\begin{array}{c}Q \text { continuous and } n \text { discrete } \\
\text { Solution procedure for Case 1 }\end{array}$ & $\begin{array}{c}Q \text { and } n \text { discrete } \\
\text { Solution procedure for Case 2 }\end{array}$ & Lower bound \\
\hline Instance 1 & $Q=1735.12899$ & $Q=1735$ & LB $=485540.6485389$ for Case 1 \\
Example from Chiu et al. [7] & $n=3$ & $n=3$ & (given (13)) \\
and Chiu et al. [8] & $E[T C U]=485540.66029$ & $E[T C U]=485540.66058$ & LB $=485540.66029$ for Case 2 \\
\hline Instance 2 & $Q=2385.34012$ & $Q=2385$ & LB $=425847.28209$ for Case 1 \\
Special Case & $n=5$ & $n=5$ & (given (13)) \\
$(x=0$ then $E(x)=0)$ & $E[T C U]=425862.39472$ & $E[T C U]=425862.39559$ & LB = 425862.39472 for Case 2 \\
\hline
\end{tabular}

Note that Chiu et al. [7] reported for the special case that the replenishment lot size is 2018 units and that the number of shipments is 3 with a total cost of 427938. The reader can notice that the solution for the special case reported by Chiu et al. [7] is erroneous.

\section{Conclusions}

The main and new contribution of this paper is to present two easy-to-apply solutions procedures to determine jointly both the optimal replenishment lot size and the optimal number of shipments for the inventory model proposed by Chiu et al. [7] and Chiu et al. [8]. The solution procedures are developed for solving two cases: Case 1: the replenishment lot size (Q) being continuous and the number of shipments $(n)$ being discrete and Case 2: the replenishment lot size (Q) being discrete and the number of shipments $(n)$ being discrete. The proposed solution procedures are simple and require no tedious computational effort. Furthermore, the proposed solution procedures discriminate between the situation in which there is a single solution and when there are two solutions for each discrete variable. Chiu et al. [7] considered the decisions variables ( $Q$ and $n$ ) to be continuous and then round up the decision variable number of shipments $(n)$. This could give us a nonoptimal value for $n$. Our paper improves and complements Chiu et al. [7] and Chiu et al. [8] research works since it treats both variables according to their nature. The readers who are interested in this paper may also refer to the research works of Cárdenas-Barrón et al. [21-23]. Finally, this paper can be extended in several ways. For example, 
one can consider stochastic demand, shortages with full or partial backordering, and deteriorating rate, just to name a few future researches.

\section{Conflict of Interests}

The authors declare that there is no conflict of interests regarding the publication of this paper.

\section{Acknowledgments}

This research was supported by the Tecnológico de Monterrey Research Group in Industrial Engineering and Numerical Methods 0822B01006. The research for the third author was supported by the Iran National Science Foundation (INSF), Fund no. INSF-93040840.

\section{References}

[1] F. W. Harris, "How many parts to make at once," Factory: The Magazine of Management, vol. 10, no. 2, pp. 135-136, 152, 1913.

[2] E. W. Taft, "The most economical production lot," The Iron Age, vol. 101, pp. 1410-1412, 1918.

[3] A. A. Taleizadeh, L. E. Cárdenas-Barrón, J. Biabani, and R. Nikousokhan, "Multi products single machine EPQ model with immediate rework process," International Journal of Industrial Engineering Computations, vol. 3, no. 2, pp. 93-102, 2012.

[4] Y.-C. Chen, "An optimal production and inspection strategy with preventive maintenance error and rework," Journal of Manufacturing Systems, vol. 32, no. 1, pp. 99-106, 2013.

[5] B. Pal, S. S. Sana, and K. Chaudhuri, "A mathematical model on EPQ for stochastic demand in an imperfect production system," Journal of Manufacturing Systems, vol. 32, no. 1, pp. 260-270, 2013.

[6] L. E. Cárdenas-Barrón, K.-J. Chung, and G. Treviño-Garza, "Celebrating a century of the economic order quantity model in honor of Ford Whitman Harris," International Journal of Production Economics, vol. 155, pp. 1-7, 2014.

[7] S. W. Chiu, H.-D. Lin, M.-F. Wu, and J.-Ch. Yang, "Determining replenishment lot size and shipment policy for an extended EPQ model with delivery and quality assurance issues," Scientia Iranica, Transaction E: Industrial Engineering, vol. 18, no. 6, pp. 1537-1544, 2011.

[8] Y.-S. P. Chiu, H.-D. Lin, and H.-H. Chang, "Determination of production-shipment policy using a two-phase algebraic approach," Maejo International Journal of Science and Technology, vol. 6, no. 1, pp. 119-129, 2012.

[9] J. García-Laguna, L. A. San-José, L. E. Cárdenas-Barrón, and J. Sicilia, "The integrality of the lot size in the basic EOQ and EPQ models: applications to other production-inventory models," Applied Mathematics and Computation, vol. 216, no. 5, pp. 16601672, 2010.

[10] R. W. Grubbström, "Material requirements planning and manufacturing resource planning," in International Encyclopedia of Business and Management, M. Warner, Ed., Routledge, London, UK, 1996.

[11] M. Omar, M. B. Zubir, and N. H. Moin, "An alternative approach to analyze economic ordering quantity and economic production quantity inventory problems using the completing the square method," Computers and Industrial Engineering, vol. 59, no. 2, pp. 362-364, 2010.
[12] H. M. Wee, S. L. Chung, and P. C. Yang, "Technical notea modified EOQ model with temporary sale price derived without derivatives," The Engineering Economist, vol. 48, no. 2, pp. 190-195, 2003.

[13] P. C. Yang and H. M. Wee, "The economic lot size of the integrated vendor-buyer inventory system derived without derivatives," Optimal Control Applications and Methods, vol. 23, no. 3, pp. 163-169, 2002.

[14] G. Treviño-Garza, K. K. Castillo-Villar, and L. E. CárdenasBarrón, "Joint determination of the lot size and number of shipments for a family of integrated vendor-buyer systems considering defective products," International Journal of Systems Science, vol. 46, no. 9, pp. 1705-1716, 2014.

[15] A. A. Taleizadeh, S. S. Kalantari, and L. E. Cárdenas-Barrón, "Determining optimal price, replenishment lot size and number of shipments for an EPQ model with rework and multiple shipments," Journal of Industrial and Management Optimization, vol. 11, no. 4, pp. 1059-1071, 2015.

[16] S. S. Sana, "An economic order quantity model for nonconforming quality products," Service Science, vol. 4, no. 4, pp. 331-348, 2012.

[17] B. Pal, S. S. Sana, and K. Chaudhuri, "A stochastic inventory model with product recovery," CIRP Journal of Manufacturing Science and Technology, vol. 6, no. 2, pp. 120-127, 2013.

[18] B. Pal, S. S. Sana, and K. Chaudhuri, "Maximising profits for an EPQ model with unreliable machine and rework of random defective items," International Journal of Systems Science, vol. 44, no. 3, pp. 582-594, 2013.

[19] M. Das Roy, S. S. Sana, and K. Chaudhuri, "An economic production lot size model for defective items with stochastic demand, backlogging and rework," IMA Journal of Management Mathematics, vol. 25, no. 2, pp. 159-183, 2014.

[20] L. E. Cárdenas-Barrón, "The derivation of EOQ/EPQ inventory models with two backorders costs using analytic geometry and algebra," Applied Mathematical Modelling, vol. 35, no. 5, pp. 2394-2407, 2011.

[21] L. E. Cárdenas-Barrón, A. A. Taleizadeh, and G. Treviño-Garza, "An improved solution to replenishment lot size problem with discontinuous issuing policy and rework, and the multi-delivery policy into economic production lot size problem with partial rework," Expert Systems with Applications, vol. 39, no. 18, pp. 13540-13546, 2012.

[22] L. E. Cárdenas-Barrón, B. Sarkar, and G. Treviño-Garza, “An improved solution to the replenishment policy for the EMQ model with rework and multiple shipments," Applied Mathematical Modelling, vol. 37, no. 7, pp. 5549-5554, 2013.

[23] L. E. Cárdenas-Barrón, B. Sarkar, and G. Treviño-Garza, “Easy and improved algorithms to joint determination of the replenishment lot size and number of shipments for an EPQ model with rework," Mathematical and Computational Applications, vol. 18, no. 2, pp. 132-138, 2013. 


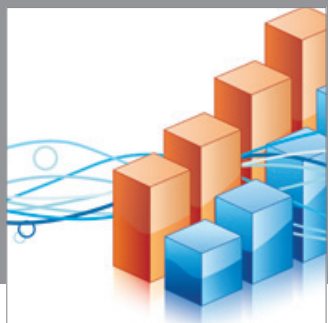

Advances in

Operations Research

mansans

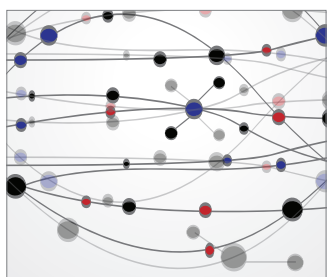

The Scientific World Journal
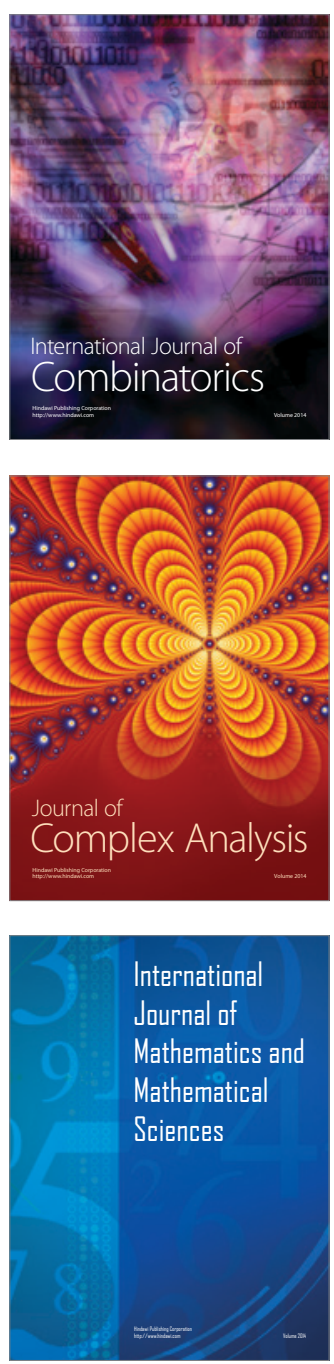
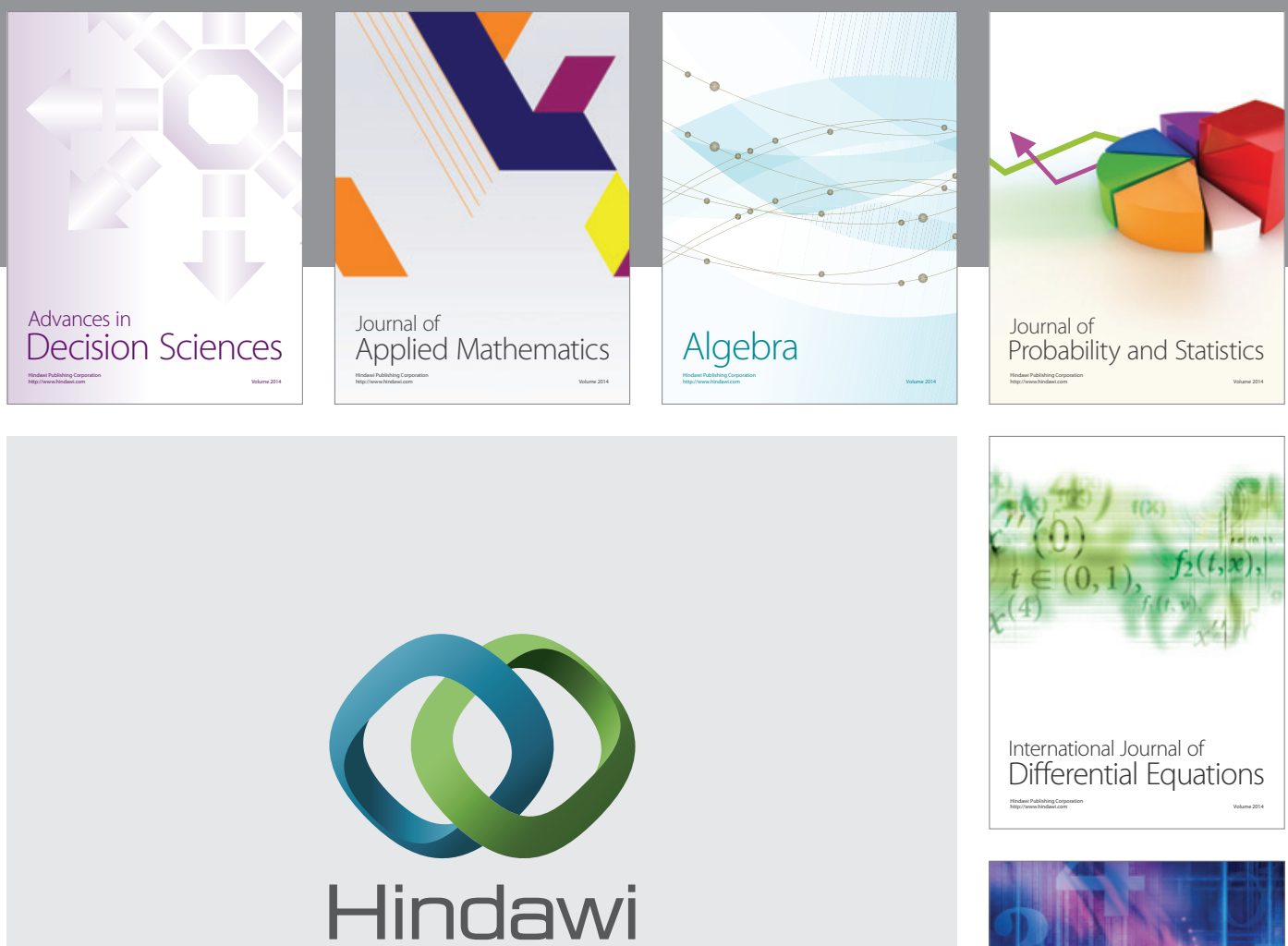

Submit your manuscripts at http://www.hindawi.com
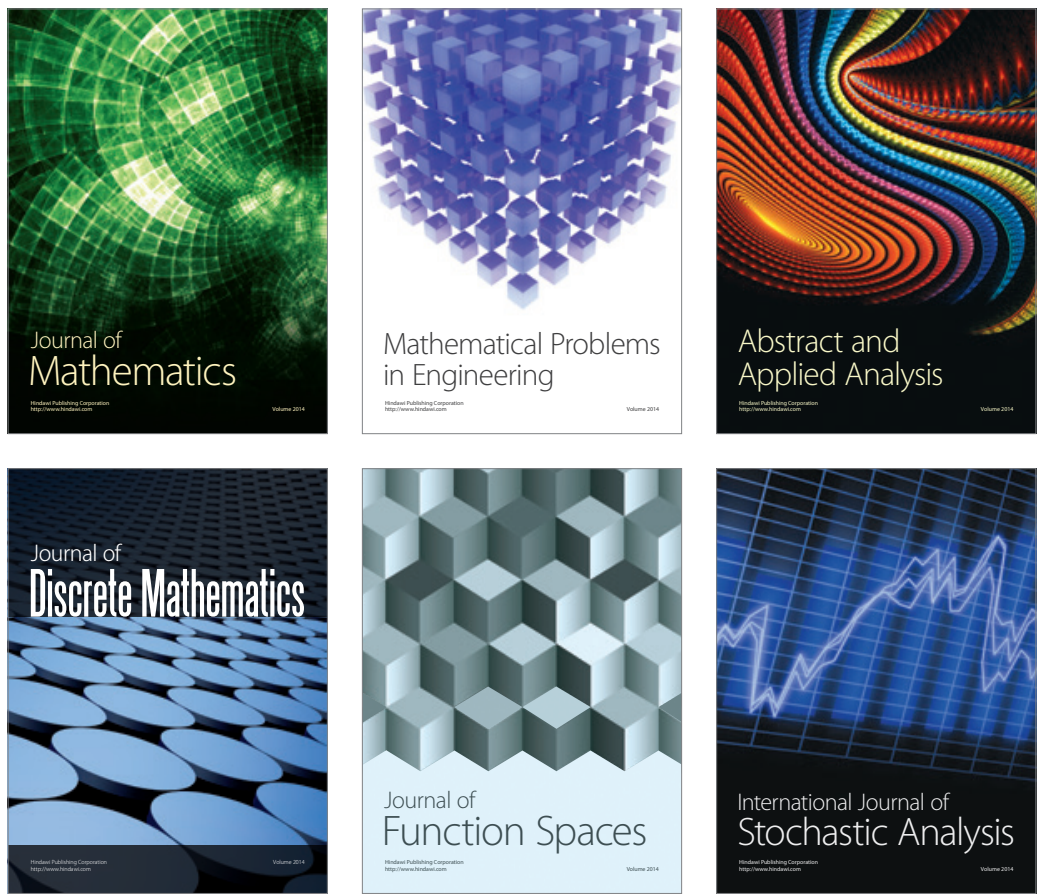

Journal of

Function Spaces

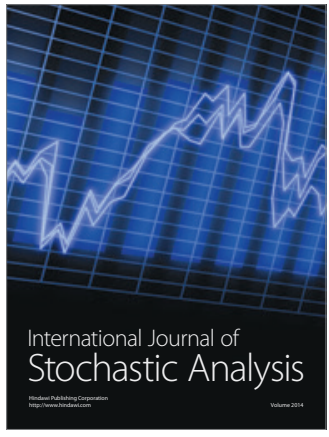

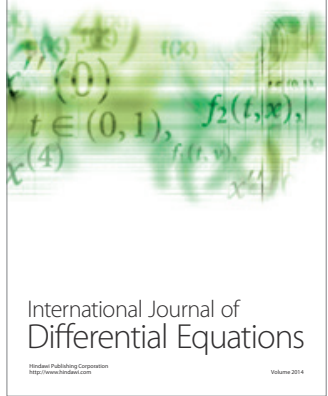
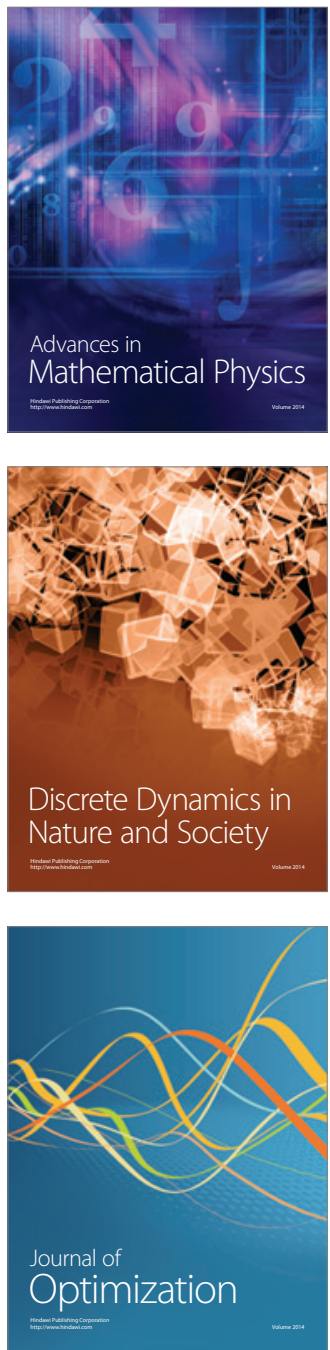